\title{
Productividad científica y evolución de las tesis doctorales en Educación Superior: análisis por género y temáticas
}

\author{
Camino Ferreira \\ Departamento de Psicología, Sociología y Filosofía, Facultad de Educación, Universidad de León. \\ Correo-e: camino.ferreira@unileon.es | ORCID iD: https://orcid.org/0000-0001-8131-0825
}

Recibido: 13-09-19; 2a versión: 25-10-19; Aceptado: 31-12-19.

Cómo citar este artículo/Citation: Ferreira, C. (2020). Productividad científica y evolución de las tesis doctorales en Educación Superior: análisis por género y temáticas. Revista Española de Documentación Científica, 43 (4), e278. https://doi.org/10.3989/ redc.2020.4.1711

Resumen: El objetivo de este estudio es analizar la productividad científica sobre Educación Superior, profundizando en los parámetros de las tesis doctorales sobre este campo atendiendo a variables bibliométricas e identificando los tópicos de las tesis doctorales y las tendencias en Educación Superior en la consecución del título de Doctor. Este estudio es de carácter descriptivo longitudinal, retrospectivo y bibliométrico de la producción científica en tesis doctorales defendidas en las universidades españolas sobre Educación Superior y registradas en la base de datos TESEO desde su primera publicación, 1976, hasta el curso académico 2018-2019. Las variables analizadas en el estudio ponen de manifiesto las diferencias entre hombres y mujeres en el ámbito académico, especialmente a la hora de ostentar el cargo de presidenta de tribunal de tesis doctoral. Los resultados reflejan que este campo ha sufrido un proceso de consolidación con un crecimiento lineal y constante en la producción de tesis doctorales.

Palabras clave: Educación Superior; tesis doctoral; bibliometría; producción científica; indexación; documentación científica.

\section{Scientific productivity and evolution of doctoral theses in Higher Education: analysis by gender and topics}

\begin{abstract}
The aim of this study is to analyze the scientific productivity of Higher Education, deepening in the doctoral theses parameters in this field, taking into account bibliometric variables and identifying the topics of the doctoral theses and trends in Higher Education in the attainment of the PhD degree. This study is descriptive longitudinal, retrospective and bibliometric of scientific production in doctoral theses on Higher Education defended in Spanish universities and registered in the TESEO database from its first publication, 1976, to the academic year 2018-2019. The analyzed variables in the study show he differences between men and women in the academic field, especially when holding the position of president of the doctoral thesis court. The results reflect that this field has undergone a process of consolidation with a linear and constant growth in the production of doctoral theses.
\end{abstract}

Keywords: Higher Education; doctoral thesis; bibliometrics; scientific production; indexing; scientific documentation.

Copyright: ( 2020 CSIC. Este es un artículo de acceso abierto distribuido bajo los términos de la licencia de uso y distribución Creative Commons Reconocimiento 4.0 Internacional (CC BY 4.0). 


\section{INTRODUCCIÓN}

La tesis doctoral es considerada por la normativa actual como un trabajo original de investigación que es desarrollado por un estudiante en cualquier campo de conocimiento. Este trabajo culmina con su defensa ante un tribunal y debe incorporar resultados originales de investigación, capacitando al doctorando para trabajar de forma autónoma en I+D+i. Por ello, las tesis doctorales se pueden considerar un buen referente para conocer cuáles son las líneas y las tendencias que se están llevando a cabo en el ámbito científico en la universidad, así como para conocer la estructura social de la investigación a nivel universitario (Delgado López-Cózar y otros, 2006).

La universidad posee el reconocimiento de ser aquel espacio en el que se desarrollan capacidades críticas e innovadoras basadas en las necesidades de la sociedad, en el que se desarrolla investigación de calidad que de respuesta a los problemas que la sociedad plantea. En este sentido, la formación de los doctores debe estar dirigida a no ser sólo un requisito para trabajar en la institución universitaria, "sino en formar investigadores, para este siglo XXI, en competencias que cubran espacios profesionales nuevos y den respuestas a los avances que la sociedad está demandando" (Hernández Pina y Díaz Martínez, 2010).

El interés por la producción científica en cualquier ámbito y tipo de documento ha fortalecido los estudios basados en análisis bibliométricos. Estos trabajos nos aportan información acerca de cómo es la situación actual y longitudinal de las áreas de conocimiento, en este caso, en las universidades y en las tesis doctorales como campo de estudio. Según Sánchez Jiménez y otros (2017), la producción existente de tesis doctorales desde 1995 hasta 2014 es proporcional a la producción científica indexada en bases de datos de carácter internacional. Esta proporción se da tanto en crecimiento a lo largo del tiempo como en la distribución entre las universidades españolas.

Las tesis doctorales han sido objeto de estudio de numerosos artículos científicos cuyo objetivo ha sido analizar la productividad de las universidades. Este método se ha llevado a cabo en diferentes áreas de conocimiento como en la ética de los medios de comunicación (Díaz Campo, 2018), en fotografía (Olivera Zaldua y otros, 2016), en geografía (De Lázaro y Torres, 2002), en internet (Díaz Campo, 2014), en psicología del deporte (Olmedilla y otros, 2017), en psiquiatría (Moyano y otros, 2006), o en medicina de urgencias y emergencias (FernándezGuerrero, 2015). En el ámbito educativo también nos encontramos este tipo de estudios bibliométricos (Moreno-Fernández y Moreno-Crespo, 2016), en algunos casos para verificar si la serie temporal se ajusta parcialmente a modelos de crecimiento de la ciencia (Fernández-Bautista y otros, 2014) o para analizar temas específicos, especialmente en tutoría y orientación (Ferreira-Villa y otros, 2013; Gómez, 2016; Salgado-Orellana y otros, 2018).

La producción de tesis doctorales se ve afectada por diversos factores, tales como el proceso de adaptación al Espacio Europeo de Educación Superior (EEES) que han vivido las universidades (Hernández Pina y Díaz Martínez, 2010), la introducción de las Tecnologías de la Información y las Comunicaciones (TIC), así como de la introducción al mundo del acceso abierto (Fuentes Pujol y Arguimbau Vivó, 2010), que ha permitido el acceso inmediato y sin restricciones a material digital académico y científico, incluyendo artículos de revistas científicas y tesis doctorales. Este trabajo parte de la concepción de que una elevada producción de tesis doctorales puede ser utilizada como un indicio positivo del desempeño científico de la universidad (Sánchez Jiménez y otros, 2017). Además, si analizamos las tesis doctorales en una disciplina, en este caso la Educación Superior, "es una buena aproximación al análisis de ciertos perfiles de la misma: los referidos a las líneas de investigación, lo que podríamos llamar el estado de la investigación y los relativos a ciertos aspectos institucionales" (Jiménez-Contreras y otros, 2014). Por ello, en este artículo se presenta información relevante sobre el análisis de la productividad en este ámbito de la educación, cuya aportación puede ser relevante para diferentes colectivos interesados como académicos, investigadores, profesionales del sector, así como instituciones universitarias.

Este estudio caracteriza la producción española adscrita al descriptor "Educación Superior" en tesis doctorales. Por lo tanto, se analiza la productividad científica en tesis doctorales sobre Educación Superior, cuantificándola y trazándola cronológicamente en función de diferentes variables, con el propósito de responder a dos objetivos:

- Conocer los parámetros de las tesis doctorales sobre Educación Superior atendiendo a variables bibliométricas.

- Identificar los tópicos de las tesis doctorales y las tendencias en Educación Superior en la consecución del título de Doctor.

\section{MATERIAL Y MÉTODO}

Este estudio es de carácter descriptivo longitudinal, retrospectivo y bibliométrico de la producción científica en tesis doctorales sobre Educación Superior en España a través de la búsqueda avanzada en la base de datos TESEO desde su primera publicación, en 1976, hasta el curso académico 2018-2019. 
TESEO es una base de datos de literatura gris editada por el Ministerio de Educación, concretamente de las Tesis Doctorales defendidas en las universidades españolas desde el año 1976. La información es proporcionada al Consejo de Coordinación Universitaria por las comisiones de doctorado de las diferentes universidades. La base de datos recoge la información de cada tesis doctoral a través de una ficha de tesis y ficheros asociados, validados por la universidad correspondiente. Debido a este hecho, esta base de datos presenta algunas limitaciones, puesto que puede producirse una falta de información si no es remitida por las universidades, produciéndose en otros casos retraso en la incorporación de la misma o falta de información descriptiva de la tesis doctoral a registrar. A pesar de ello, se ha seleccionado esta base de datos, puesto que, además de ser la principal herramienta que incluye mayores registros de este tipo de documento con mayor recorrido cronológico, es la empleada en la realización de estudios de este corte, considerándose la base de datos más completa en tesis doctorales de las universidades españolas.

La metodología empleada ha seguido cuatro fases principalmente: búsqueda documental, registro de información, análisis de datos, y redacción del informe.

\subsection{Descripción de las unidades de análisis}

El objeto de estudio de esta investigación se centra en las fuentes primarias, tesis doctorales, sobre Educación Superior. La búsqueda se realizó al finalizar el curso académico 2018-2019 en la base de datos TESEO mediante la palabra clave "Educación Superior" en el título de la tesis doctoral y como descriptor, según el Tesauro incluido en la base de datos (código 580208). Este descriptor pertenece al grupo de Educación-Sistemas y niveles de enseñanza en el Tesauro de la UNESCO, en la categoría de Niveles Educativos, Grados y Organizaciones. Se ha considerado este criterio por ser normalizado y consistente en una nomenclatura internacional. La elección de este descriptor viene justificada porque las tesis doctorales que se encuentran en este campo de estudio implican un posicionamiento por parte del autor/investigador, debiendo etiquetarse con dicho código, por ello se han descartado las que no han sido consideradas de esta manera (Díaz Campo, 2014).

El número de registros encontrados en la búsqueda avanzada ha sido de 149, que responden a todas las tesis doctorales desde 1976 hasta el curso académico 2018-2019, que tratan de Educación Superior y así lo reflejan en sus títulos y descriptores.

\subsection{Descripción de las variables}

El análisis realizado se compone de las siguientes variables $(\mathrm{N}=17)$ :

- Universidad

- Comunidad Autónoma

- Departamento

- Área temática

- Rama de conocimiento

- Año de lectura

- Disponibilidad de la tesis doctoral a texto completo

- Mención Europea o Mención Internacional

- Programa de doctorado

- No de directores de la tesis doctoral

- No miembros del tribunal de la tesis doctoral

- Categoría de los miembros del tribunal evaluador

- Género del autor/a de la tesis doctoral

- Género de los directores de la tesis doctoral

- Género de los miembros del tribunal de la tesis doctoral

- Género de los miembros del tribunal evaluador de la tesis doctoral según el cargo desarrollado en el mismo

\section{- Descriptores}

La base de datos TESEO no dispone de una exportación de los registros a ningún gestor bibliográfico, así como la descarga de los mismos de forma automática. Por ello, se han registrado cada uno de los campos de la base de datos en un registro de Excel (Título, Autor, Universidad, Departamento, Fecha de Lectura, Mención Europeo/ Mención Internacional, Programa de doctorado, Dirección, Tribunal, Descriptores, Disponibilidad de la Tesis Doctoral, Resumen), realizando los análisis estadísticos correspondientes.

Una vez realizado el volcado de datos, se han realizado análisis estadísticos univariantes y descriptivos, así como análisis de asociación entre las variables a través del índice de significatividad $(p<0.005)$ de la prueba Chi-Cuadrado. Para el análisis de contenido de los títulos de las tesis doctorales se ha utilizado el programa de análisis cualitativo MAXQDA 2018.

\section{RESULTADOS}

De las 252.955 tesis doctorales publicadas en la base de datos TESEO desde 1976 hasta el curso académico 2018-2019, hay 149 que tratan direc- 
tamente la Educación Superior como objeto de estudio. A continuación, se presentan los resultados analizados de los registros identificados en función de las variables definidas.

A nivel institucional, las universidades españolas con mayor productividad en tesis doctorales sobre Educación Superior en el periodo analizado son: la Universidad de Granada $(n=19)$, seguida de la Universidad Autónoma de Barcelona $(n=13)$, la Universidad de Alicante $(n=12)$ y la Universidad de Salamanca $(n=9)$. Un número significativo de ellas también son defendidas en la Universidad Autónoma de Madrid, la Universidad de A Coruña, la Universidad de León y la Universidad de Murcia, con 6 tesis por universidad, y la Universidad de Málaga y la Universidad de Sevilla, con 5. En la Tabla I se puede observar la distribución de las tesis doctorales en Educación Superior por universidad durante el periodo analizado, agrupadas por cuatrienios.

A nivel de Comunidad Autónoma, hay que tener en cuenta el número de universidades con las que cuenta, así como la financiación que reciben cada una de ellas. Esto es debido a que la producción científica se relaciona con los recursos económicos disponibles, influyendo la cantidad de los mismos, así como su gestión. En la Figura 1 se puede ver la distribución de las tesis doctorales defendidas en las universidades españolas por Comunidad Autónoma, destacando Cataluña y Andalucía como se ha comprobado en anteriores estudios sobre producción y eficiencia en investigación en las universidades españolas (Buela-Casal y otros, 2014; Buela-Casal y otros, 2015).

Los departamentos en los que se encuentran adscritos los programas de doctorado en los que se han desarrollado estas tesis, son numerosos y variados en denominación $(n=61)$. Muchos de ellos pertenecen a departamentos con áreas temáticas conjuntas (por ejemplo, Psicología Básica, Evolutiva y de la Educación; Psicología, Sociología y Filosofía; Filosofía y Métodos de Investigación en Educación), por ello, se han analizado teniendo en cuenta las áreas temáticas de la Agencia Nacional de Evaluación y Prospectiva (ANEP), las cuales se agrupan en 25 Áreas Temáticas dentro de las que se encuadran diferentes Áreas de Conocimiento. Asimismo, se han clasificado los departamentos según las ramas de conocimiento que sigue la Agencia Nacional de Evaluación de la Calidad y Acreditación (ANECA): Artes y Humanidades, Ciencias, Ciencias de la Salud, Ciencias Sociales y Jurídicas, e Ingeniería y Arquitectura (ver Tabla II). El $80 \%$ de ellas pertenecen a la rama de Ciencias Sociales y Jurídicas, existiendo un pequeño porcentaje en Ingeniería y Arquitectura y en Arte y Humanidades. Por ello, las principales áreas en las que se enmarcan las tesis doctorales en Educación Superior son en el Área de Ciencias de la Educación $(n=23)$, destacando los departamentos de Didáctica y Organización Escolar y Métodos de Investigación y Diagnóstico en Educación; el Área de Ciencias Sociales $(n=15)$, con departamentos como Biblioteconomía y Documentación o Antropología Social; el Área de Economía $(n=8)$, especialmente el departamento de Organización de Empresas; y el Área de Psicología $(n=4)$ en el que destaca el departamento con la misma denominación.

A nivel cronológico, hasta los años 2000 apenas se defendía una tesis por año y aunque a partir de este año aumenta gradualmente, se mantiene por debajo de 7 anuales (la media en los 43 años es de 3,5 tesis/año). Es en el año 2012 cuando la productividad en Educación Superior ha aumentado considerablemente hasta 2018 (ver Figura 2). Este hecho puede haberse visto impulsado por la implantación cinco años antes del EEES en España, a través del Real Decreto 1393/2007, de 29 de octubre, por el que se establece la ordenación de las enseñanzas universitarias oficiales. Aunque hay periodos de años sin defender tesis doctorales en Educación Superior y no existe un número estable de tesis por curso académico, lo cierto es que la tendencia al crecimiento ha aumentado a través de las décadas. En los primeros años del periodo analizado correspondiente a las décadas de los años 80 y 90 se publicaron un total 6 tesis doctorales, en la década de los 2000 se publicaron un total de 19; y solamente en el año 2016 se leyeron 28, alcanzando el máximo de tesis doctorales defendidas en el periodo analizado.

El $42 \%$ de las tesis doctorales analizadas se encuentran disponibles a texto completo $(n=62)$ mientras que el $58 \%$ sólo disponen del resumen $(n=87)$. Cabe señalar que este hecho se concentra en los últimos años debido, entre otros, al artículo 14 del Real Decreto 99/2011, de 28 de enero, por el que se regulan las enseñanzas oficiales de doctorado, que indica la obligación a publicarla en los repositorios instituciones de las universidades, por exigencia asimismo de la Ley $14 / 2011$, de 1 de junio, de la Ciencia, la Tecnología y la Innovación. Este Real Decreto indica que una vez que se ha aprobado la tesis doctoral, la universidad tiene la obligación de incluirla en formato electrónico abierto en un repositorio institucional, remitiéndola asimismo al Ministerio de Educación (organismo responsable y gestor de TESEO) en formato electrónico junto con la información pertinente sobre el proceso de defensa de tesis doctoral. Esta publicación puede estar sujeta a circunstancias excepcionales como puede ser la existencia de convenios de confidencialidad con empresas o la posible creación de patentes relacionadas directamente con el contenido de la tesis doctoral. 


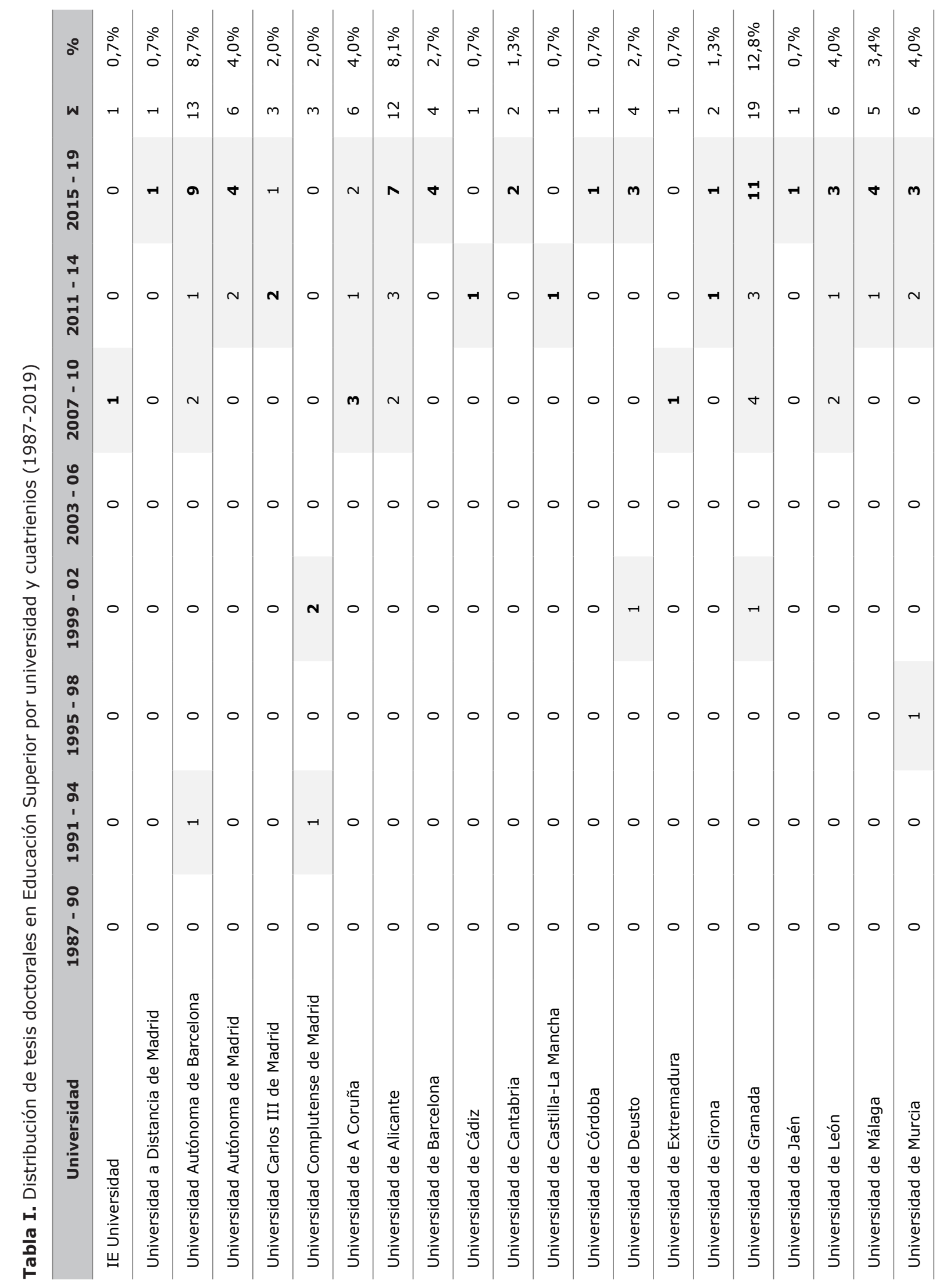




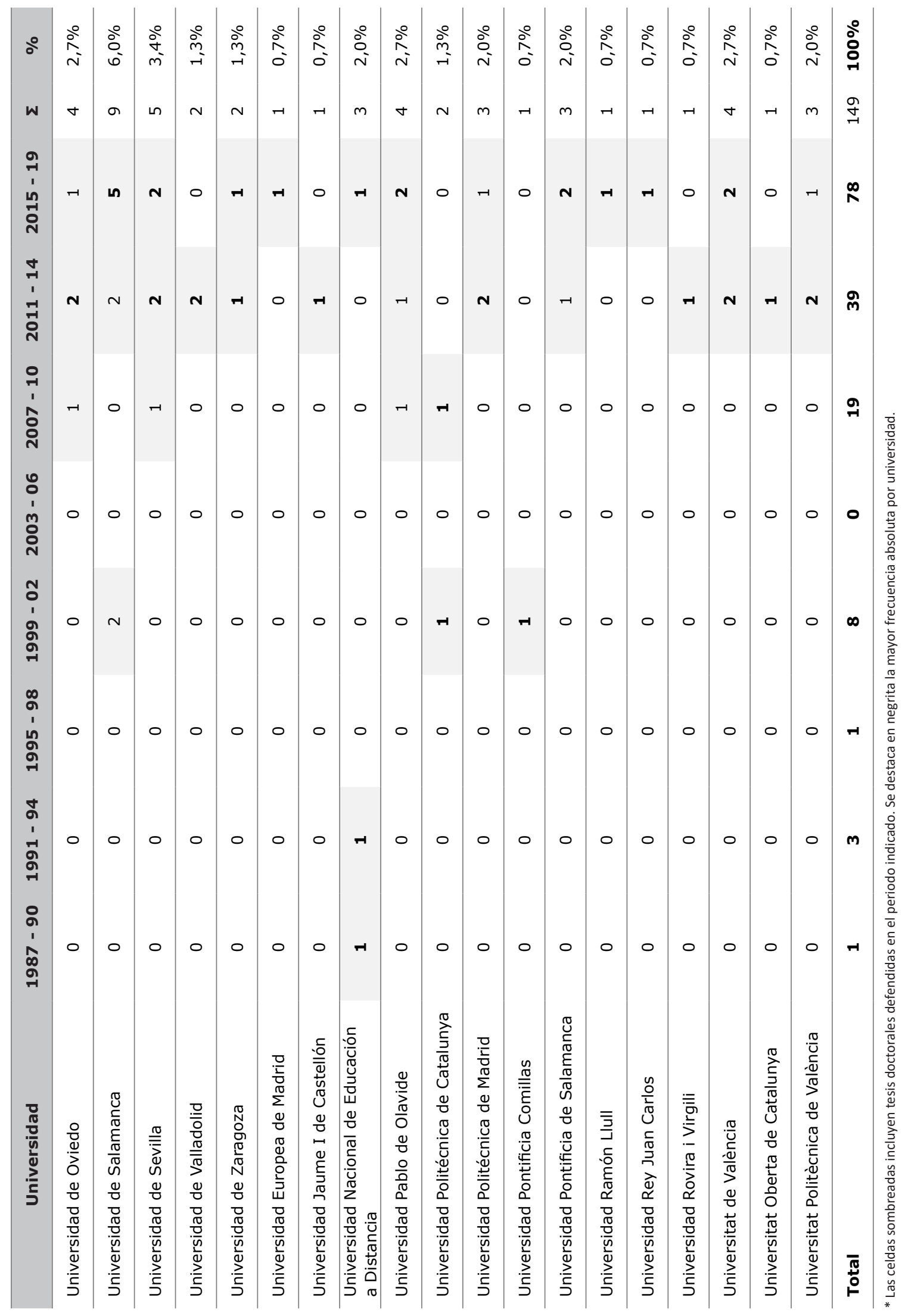


Figura 1. Número de tesis doctorales en Educación Superior por Comunidad Autónoma

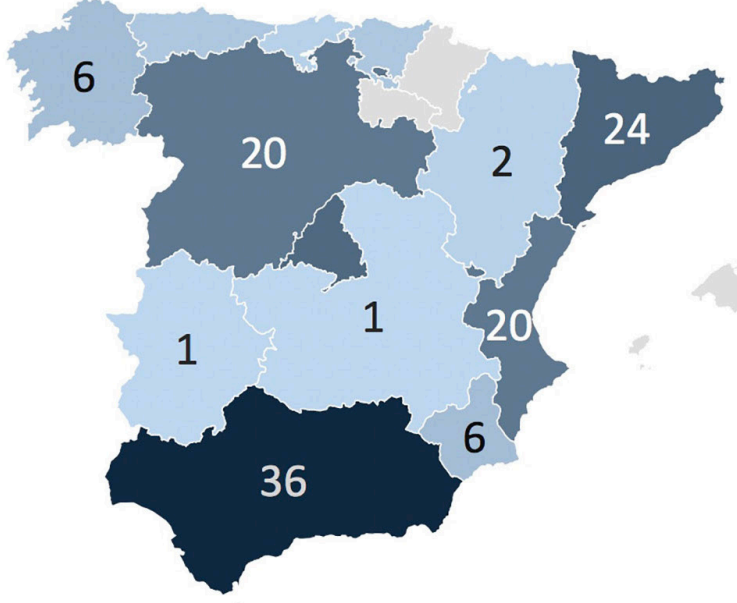

$\mathrm{N}$

Tabla II. Áreas temáticas y áreas de conocimiento de las tesis doctorales en Educación Superior

\begin{tabular}{|c|c|c|}
\hline Área Temática & $\mathbf{N}$ & $\%$ \\
\hline Área de Agricultura (AGR) & 1 & $2 \%$ \\
\hline Área de Ciencias de la Computación y Tecnología Informática (INF) & 2 & $3 \%$ \\
\hline Área de Ciencias de la tierra $(\mathrm{CT})$ & 1 & $2 \%$ \\
\hline Área de Ciencias Sociales (CS) & 15 & $25 \%$ \\
\hline Área de Derecho (DER) & 1 & $2 \%$ \\
\hline Área de Economía (ECO) & 8 & $13 \%$ \\
\hline Área de Ciencias de la Educación (EDUC) & 23 & $38 \%$ \\
\hline Área de Historia y Arte (HA) & 2 & $3 \%$ \\
\hline Área de Matemáticas (MTM) & 1 & $2 \%$ \\
\hline Área de Medicina Clínica y Epidemiología (MCLI) & 1 & $2 \%$ \\
\hline Área de Psicología (PS) & 4 & $7 \%$ \\
\hline Área de Tecnología Electrónica y de las Comunicaciones (COM) & 1 & $2 \%$ \\
\hline Sin identificar & 1 & $2 \%$ \\
\hline Total & 61 & $100 \%$ \\
\hline Rama de Conocimiento & $\mathbf{N}$ & $\%$ \\
\hline Ciencias & 0 & $0 \%$ \\
\hline Ciencias de la Salud & 1 & $2 \%$ \\
\hline Ingeniería y Arquitectura & 5 & $8 \%$ \\
\hline Ciencias Sociales y Jurídicas & 49 & $80 \%$ \\
\hline Arte y Humanidades & 5 & $8 \%$ \\
\hline Sin identificar & 1 & $2 \%$ \\
\hline Total & 61 & $100 \%$ \\
\hline
\end{tabular}


Figura 2. Evolución de las tesis doctorales en Educación Superior defendidas, disponibles y con Mención Europea o Internacional, por cuatrienios

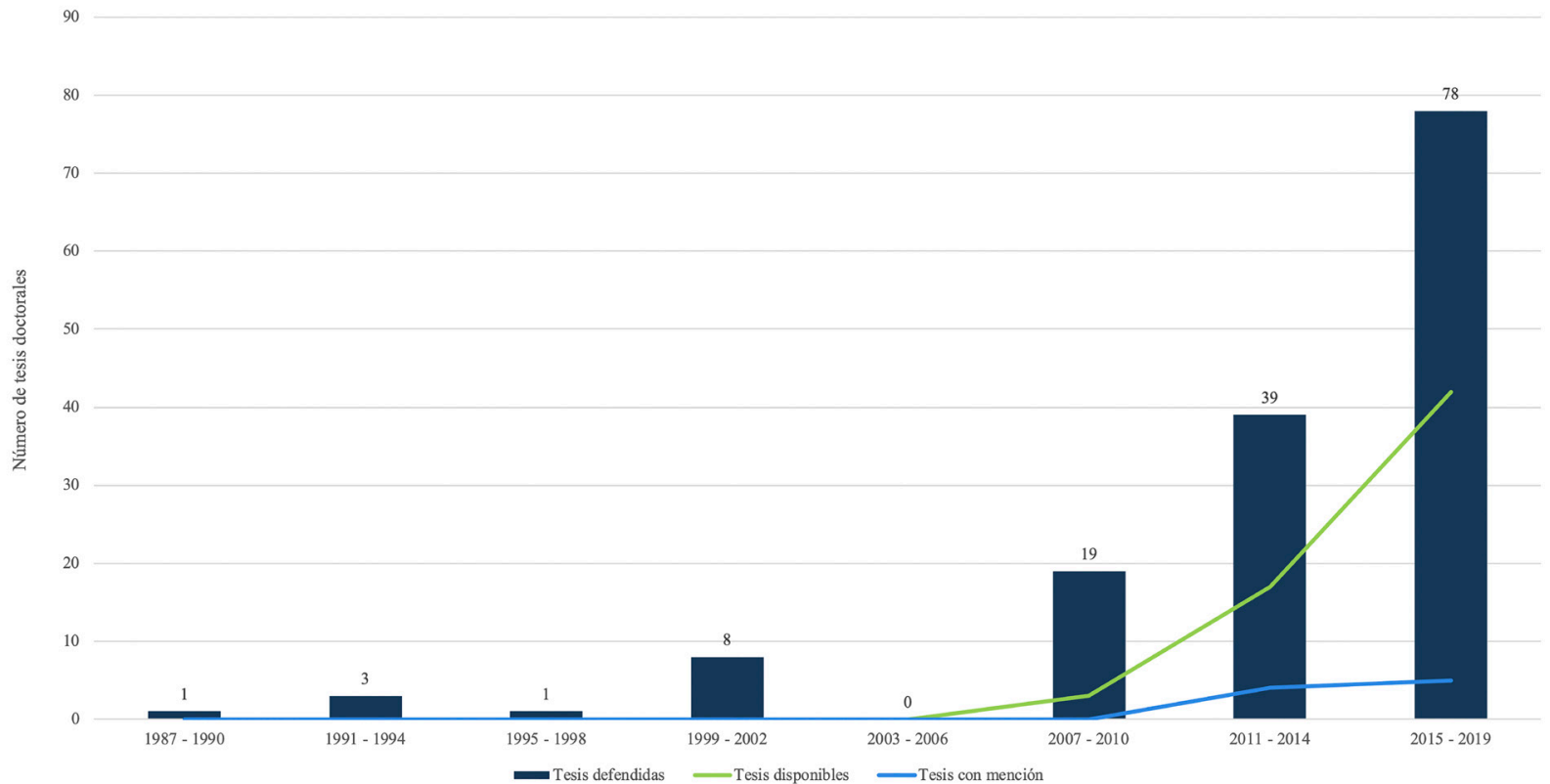

Solamente 9 de las 149 tesis doctorales disponen de Mención Europea o de Mención Internacional. Esta consideración en las tesis doctorales es relativamente reciente y se concede cuando el doctorando dispone de una serie de requisitos. Aunque en el Real Decreto 1393/2007, de 29 de octubre, por el que se establece la ordenación de las enseñanzas universitarias oficiales no se menciona esta Mención, sí que se establece la posibilidad de incluir en el título la mención de "Doctor Internacional" posteriormente, en el Real Decreto 99/2011, de 28 de enero, por el que se regulan las enseñanzas oficiales de doctorado. En esta normativa, en su artículo 15.1, se concretan las circunstancias para que se pueda optar a esta Mención y así figure en el título de Doctor, las cuales afectan a la realización de estancias en el extranjero, la redacción y presentación de parte de la tesis en otra lengua oficial o cooficial de España y a la evaluación de la tesis por expertos Doctores pertenecientes a instituciones de Educación Superior o centros de investigación no españoles. En la Figura 2 se observa que la primera tesis doctoral con esta consideración de Mención Europea o Mención Internacional aparece en el año 2012, manteniéndose constante en los últimos años analizados.

Entre los criterios de evaluación para la verificación y acreditación de los programas de doctorado también se tiene en cuenta el grado de internacionalización de los doctorados valorando los re- sultados de menciones europeas e internacionales (artículo 10.5). Esta Mención ha sido concedida en las tesis doctorales identificadas $(n=9)$ en los Programas de Doctorado en:

- Comunicación por la Universidad de Cádiz; la Universidad de Huelva; la Universidad de Málaga y la Universidad de Sevilla.

- Economía y Empresa por la Universidad Autónoma de Madrid.

- Educación y Comunicación Social por la Universidad de Málaga.

- Ciencias de la Educación por la Universidad de Granada.

- Tecnología Educativa por la Universidad de Salamanca.

- La Sociedad de la Información: Retos Sociales e Instrumentos de Análisis por la Universidad de A Coruña.

- Investigación e Innovación en Educación por la Universidad de Valladolid.

- Programa de Doctorado en Antropología y Bienestar Social por la Universidad de Granada.

- Programa de Doctorado en Fundamentos del Curriculum y Formación del Profesorado en las Áreas de Primaria y Secundaria por la Universidad de Granada. 
Un amplio número de tesis doctorales $(n=65)$ están co-dirigidas por dos o tres directores (el $89 \%$ por dos directores), pero hay mayor número de tesis dirigidas por un solo director $(n=84)$. En total han participado en la dirección de estas tesis 223 directores y directoras. En la Figura 3 se observa el promedio de directores durante el periodo analizado. Hasta el año 2000, la tendencia ha sido dirigir las tesis doctorales por un solo director. Posteriormente esta tendencia ha aumentado ligeramente, alcanzando su punto máximo en el año 2013 con 1,8 directores de media durante ese año.

En relación al número de tesis doctorales defendidas en función del género por el autor o autora de las mismas, se observa que el porcentaje es muy similar en hombres (47\%) y mujeres (53\%). Esta diferencia de género va aumentando al analizar el número de tesis dirigidas por hombres, incrementándose ligeramente en un $58 \%$ frente a un $42 \%$ por mujeres. Más acuciada es esta diferencia en relación a la presencia de mujeres en los tribu- nales de tesis doctorales. En la tabla III se muestra la distribución de los miembros de los tribunales en función del género y la categoría desempeñada en las tesis doctorales en Educación Superior (los porcentajes se calculan sobre el total de hombres y mujeres). De los 596 miembros de tribunales de las tesis doctorales objeto de estudio, solamente el $38 \%$ son mujeres $(n=229)$, contando con un $62 \%$ de hombres $(n=367)$. Si nos centramos en el rol desempeñado en los tribunales (presidente/a, vocal y secretario/a) se observa que las diferencias de género aumentan considerablemente al desempeñar un cargo con mayor categoría profesional y antigüedad. De esta forma, en primer lugar, la secretaría está repartida de forma igualitaria entre mujeres y hombres. En segundo lugar, como vocales ha actuado un $40 \%$ de mujeres y un $60 \%$ de hombres, descompensándose ligeramente. Por último, es en la presidencia de los tribunales de tesis doctorales donde son los hombres los que han sido nombrados con este cargo en un $75 \%$ frente a un $25 \%$ de mujeres.

Figura 3. Tendencia a codirigir tesis doctorales en Educación Superior (1987-2018)

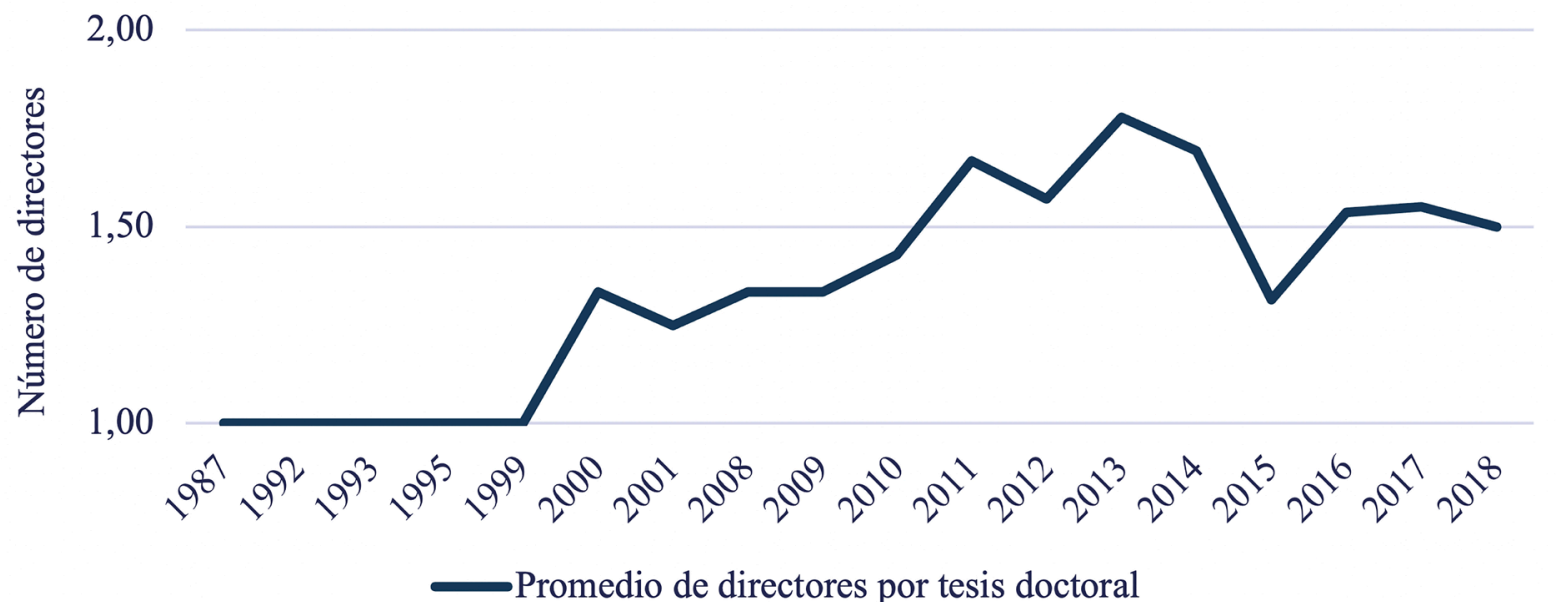

Tabla III. Distribución de los miembros de los tribunales en función del género y la categoría desempeñada en las tesis doctorales en Educación Superior

\begin{tabular}{|c|c|c|c|c|c|}
\hline & & & Mujer & Hombre & Total \\
\hline \multirow{2}{*}{\multicolumn{2}{|c|}{ Miembro de tribunal }} & $\mathrm{N}$ & 229 & 367 & 596 \\
\hline & & $\%$ & $38 \%$ & $62 \%$ & $100 \%$ \\
\hline \multirow{2}{*}{\multicolumn{2}{|c|}{ Presidente/a }} & $\mathrm{N}$ & 37 & 113 & 150 \\
\hline & & $\%$ & $25 \%$ & $75 \%$ & $100 \%$ \\
\hline \multirow{4}{*}{ Cargo } & \multirow{2}{*}{ Vocal } & $\mathrm{N}$ & 118 & 179 & 297 \\
\hline & & $\%$ & $40 \%$ & $60 \%$ & $100 \%$ \\
\hline & \multirow{2}{*}{ Secretario/a } & $\mathrm{N}$ & 74 & 75 & 149 \\
\hline & & $\%$ & $50 \%$ & $50 \%$ & $100 \%$ \\
\hline
\end{tabular}


La tendencia de la presencia de la mujer en la actividad formativa del doctorado se ha visto reflejada también en su participación en las tesis doctorales. La variable género se ha analizado a lo largo del periodo estudiado en la autoría de las tesis doctorales, en su dirección, así como en la presencia en tribunales y el cargo que se ha desarrollado en los mismos. De forma general, al analizar esta tendencia de la presencia de los hombres en la formación académica de la tesis doctoral vemos que salvo excepciones siempre es mayor que las mujeres (ver Figura 4). En el caso de la autoría, se observa que ha crecido paralelamente la cantidad de hombres y mujeres que han elaborado la tesis doctoral en este ámbito. Sin embargo, en la dirección, sí se observa cómo la presencia de los hombres ha estado prácticamente siempre por encima de la de las mujeres, a excepción de algún curso académico en los primeros años.

En el caso de la presencia en tribunales y el cargo desarrollado en ellos, se observa cómo los hombres han ocupado estos puestos, aunque la tendencia empieza a invertirse y son las mujeres quiénes tienen mayor presencia en los mismos, no siendo así en los cargos de presidencia (ver Figura 5). En relación a las variables de género y de cargo en el tribunal (secretario/a, vocal y presidente/a), en la Tabla IV se aprecia una asociación estadísticamente significativa entre las dos variables $\left(x^{2} 20.17, p<0.001\right)$. El puesto de vocal es ocupado en un porcentaje similar para hombres $(48,8 \%)$ que para mujeres $(51,5 \%)$, no ocurriendo lo mismo en el caso de secretario/a y de presidente/a. En los puestos que requieren de menor categoría y antigüedad, la presencia de las mujeres es mayor (32,3\%) que los hombres $(20,4 \%)$. Esta diferencia es aún mayor en el cargo de presidente/a donde el porcentaje de hombres $(30,8 \%)$ que han ostentado este puesto se duplica prácticamente frente al de las mujeres $(16,2 \%)$, el cual es muy bajo con respecto al resto de cargos de un tribunal de tesis doctoral. Esto se ve influido por la categoría a la que pertenecen los miembros, relacionando este dato con el obtenido por Gallego Morón (2016) en los que por cada cuatro hombres hay una mujer catedrática, por lo que tiene menos probabilidades de ocupar la presidencia del tribunal. En este caso, por cada tres hombres hay una mujer presidenta de tribunal.

Del total de las tesis doctorales analizadas $(\mathrm{N}=149), 34$ han incluido el descriptor "Educación Superior" como único descriptor (23\%). En el caso del resto de tesis doctorales, se han identificado un total de 110 descriptores diferentes a "Educación Superior". El 59\% de las tesis doctorales leídas incluyen 9 descriptores comunes (incluyendo "Educación Superior"), mientras que el $41 \%$ restante identifica 102 descriptores diferentes. Estos descriptores comunes son organización y planificación de la educación, pedagogía, organización y dirección de las instituciones educativas, evaluación de alumnos, política educativa, métodos pedagógicos, profesión

Figura 4. Presencia de mujeres y hombres en autoría y dirección de tesis doctorales en Educación Superior por cuatrienios

70

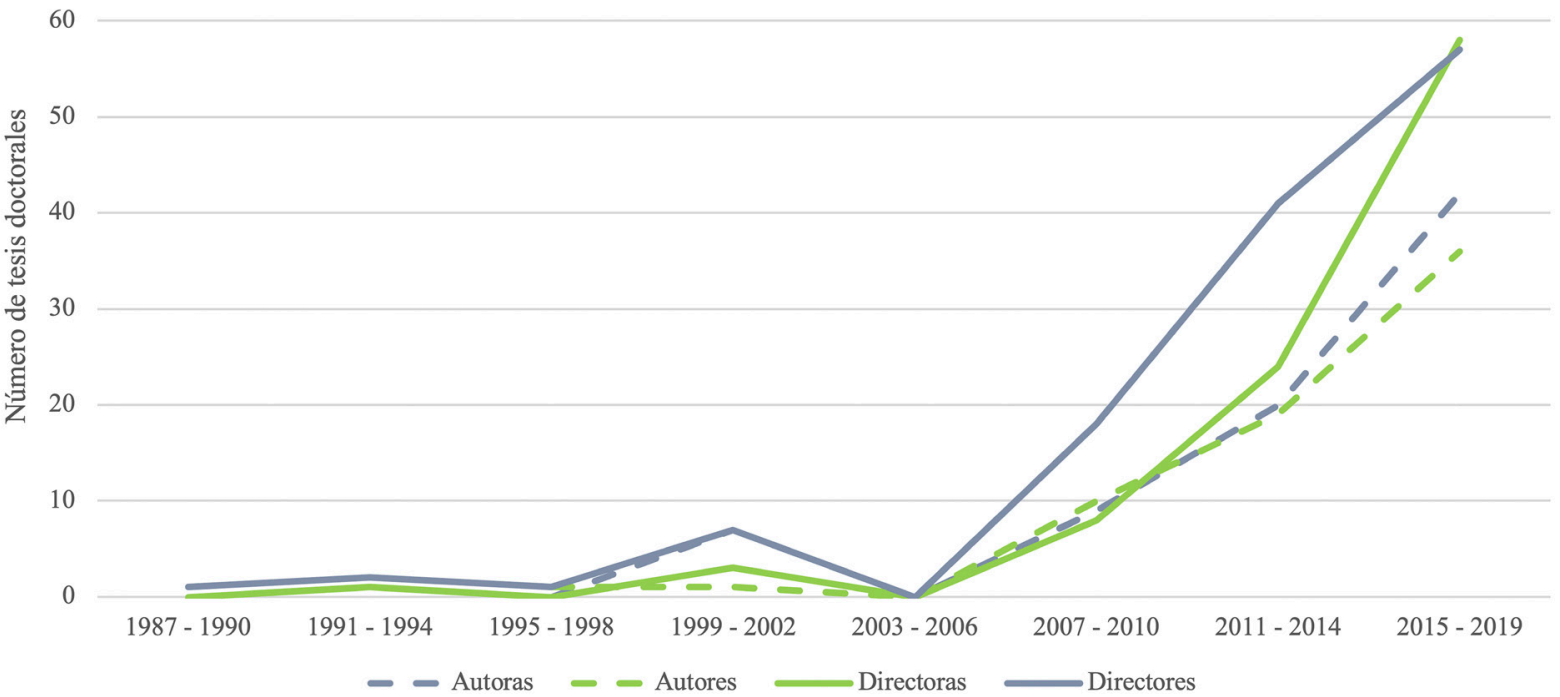


Figura 5. Presencia de mujeres y hombres en tribunales y en las presidencias de tribunal de tesis doctorales en Educación Superior por cuatrienios

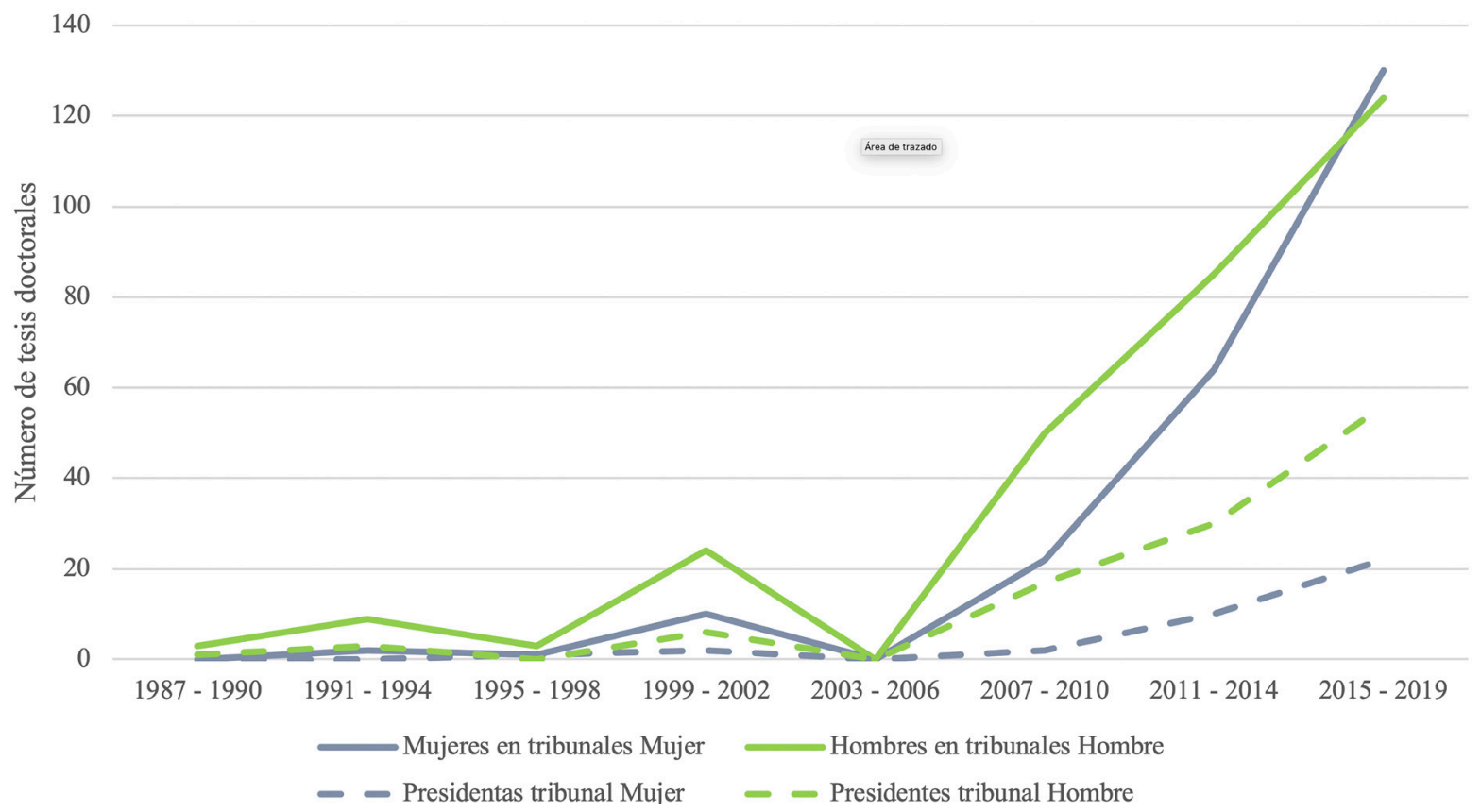

Tabla IV. Tabla de contingencia y Chi-Cuadrado de las variables sexo y cargo en el tribunal de las tesis doctorales en Educación Superior

\begin{tabular}{|c|c|c|c|c|c|}
\hline & & & Cargo & & \\
\hline & & Secretario/a & Vocal & Presidente/a & Total \\
\hline \multirow{2}{*}{ Mujer } & $\mathrm{N}$ & 74 & 118 & 37 & 229 \\
\hline & $\%$ & $32,3 \%$ & $51,5 \%$ & $16,2 \%$ & $100 \%$ \\
\hline \multirow{2}{*}{ Hombre } & $\mathrm{N}$ & 75 & 179 & 113 & 367 \\
\hline & $\%$ & $20,4 \%$ & $48,8 \%$ & $30,8 \%$ & $100 \%$ \\
\hline \multirow{2}{*}{ Total } & $\mathrm{N}$ & 149 & 297 & 150 & 596 \\
\hline & $\%$ & $25 \%$ & $49,8 \%$ & $25,2 \%$ & $100 \%$ \\
\hline \multicolumn{2}{|c|}{ Chi-Cuadrado } & \multicolumn{2}{|c|}{$X^{2} 20.17$} & \multicolumn{2}{|c|}{$\mathrm{p}<0.001$} \\
\hline
\end{tabular}

y situación del profesorado, y enseñanza con ayuda de ordenador. El análisis de estos descriptores por universidad se puede ver en la Tabla $\mathrm{V}$, destacando la Universidad Autónoma de Barcelona, la Universidad de A Coruña, la Universidad de Granada, la Universidad de León, la Universidad Nacional de Educación a Distancia y la Universidad Pontificia de Comillas, por ser aquellas universidades que presentan mayor variedad de descriptores en la temática de sus tesis doctorales en Educación Superior.

A nivel evolutivo, se observa en la Tabla VI, cómo han ido apareciendo temáticas en las tesis doctorales relacionadas con la Educación Superior a lo largo del periodo analizado. Así, descriptores como organización y planificación de la educación, pedagogía y organización y dirección de las instituciones educativas, han estado siempre presentes en estas tesis doctorales. Sin embargo, los tópicos sobre política educativa, enseñanza con ayuda de ordenador, sector de la educación y sociología de la educación, son descriptores que han aparecido en los últimos diez años por primera vez en relación con la Educación Superior. Actualmente, se observa que, dentro de la temática Educación Superior, se encuentra la tendencia a tratar este ámbito relacionado con la organización y planificación de la educación, y la política educativa, siendo los principales tópicos de interés en los últimos años. 


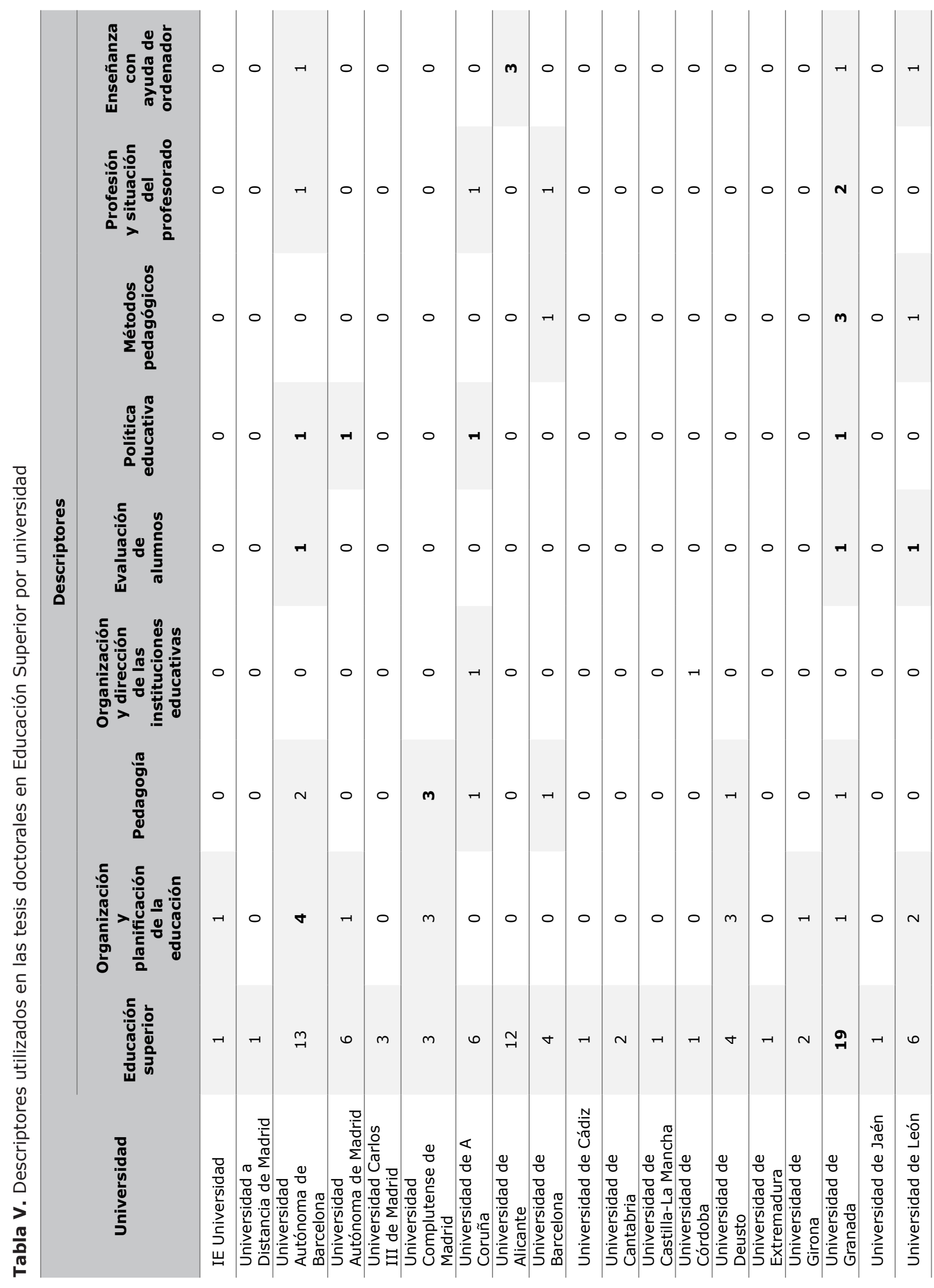




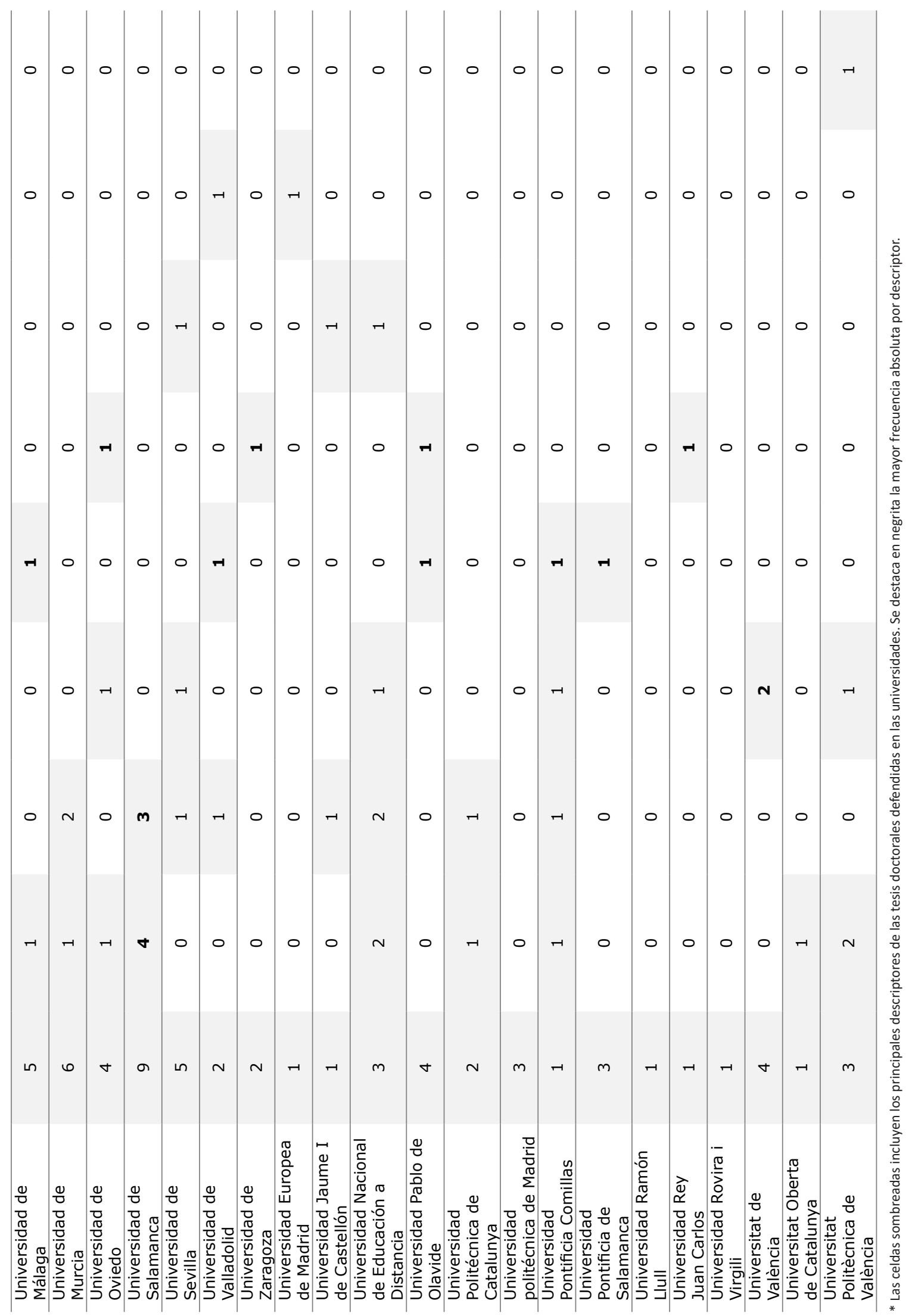




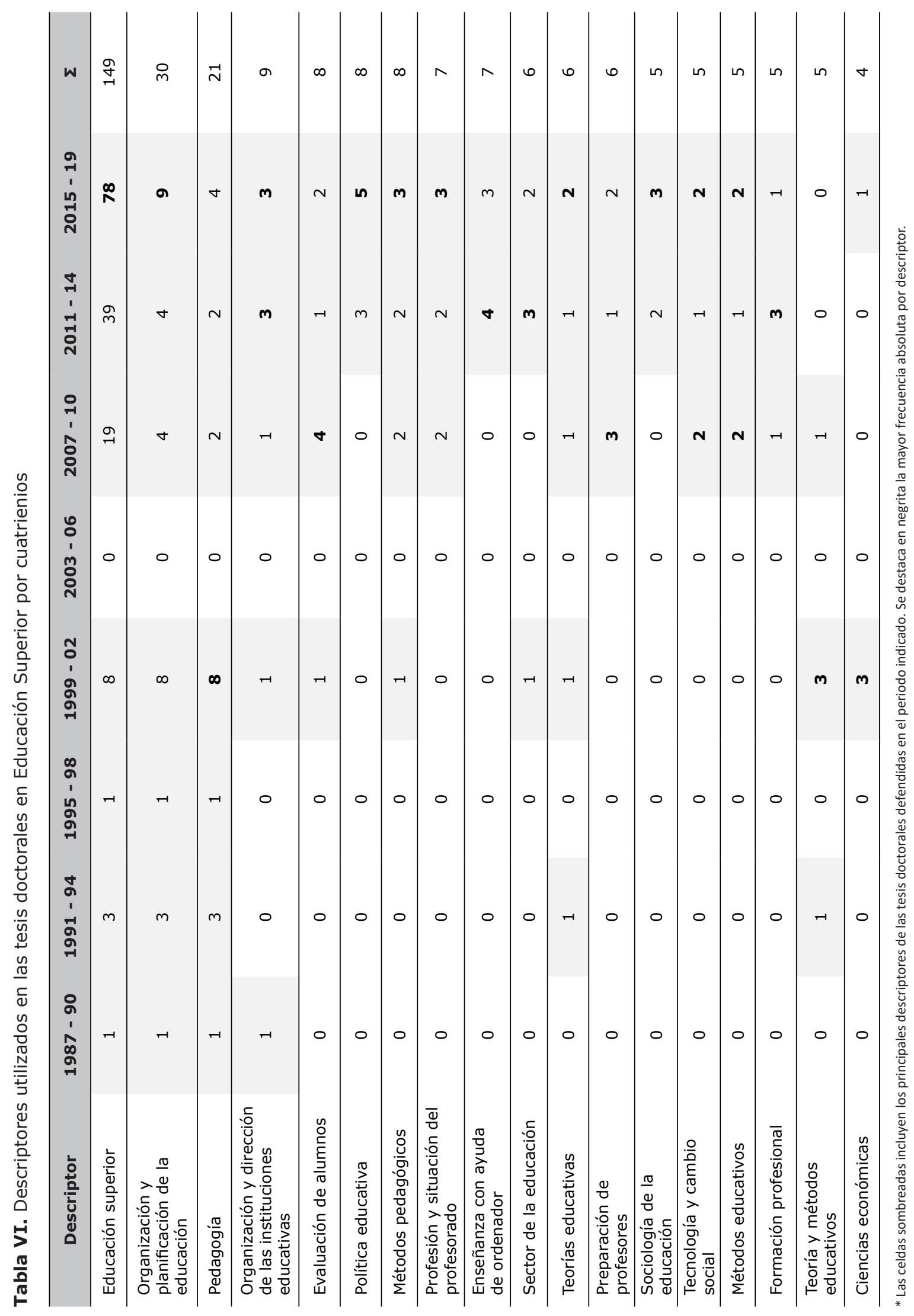


Dado el criterio de búsqueda para la selección de las fuentes de información (descriptor "Educación Superior"), las palabras "educación" y "superior" son las que con más frecuencia presentan las tesis doctorales analizadas, seguidos de "universidad" (23\%), "enseñanza" (15\%) y "estudiantes" (15\%). Destacan también términos como "espacio" y "europeo" reflejando la preocupación por el estudio del EEES (25\%). Si observamos la mayoría de los términos relacionados, hacen alusión al ámbito educativo en las universidades. De forma general, las tesis se centran en el aprendizaje y la formación (22\%), en la evaluación y calidad $(19 \%)$, en modelos (13\%) y en las instituciones $(12 \%)$. Destacan otros términos más precisos que focalizan en un ámbito en concreto como "discapacidad", "learning", "social" ( $n=8$ tesis en cada uno). En este análisis también se identifican otros países en los que se ha analizado la Educación Superior como Chile $(n=7)$, Colombia $(n=6)$, Ecuador $(n=5)$ y México $(n=5)$. La Figura 6 muestra una nube de palabras en el que se refleja la frecuencia de palabras utilizadas (a través del tamaño de las mismas) en los títulos de las tesis doctorales en Educación Superior.

\section{DISCUSIÓN Y CONCLUSIONES}

Este trabajo presenta la evolución de la producción en Educación Superior de las tesis doctorales defendidas en España desde su registro y publicación en la base de datos TESEO (1987). Del total de universidades españolas ( $\mathrm{N}=90$ según el Registro de Universidades, Centros y Títulos del Ministerio de Educación), poco más del $44 \%$ han desarrollado tesis doctorales en este sector. Destacan la Universidad de Granada y la Universidad Autónoma de Barcelona como las grandes productoras, lo que también es observado en otros estudios (MorenoFernández y Moreno-Crespo, 2016).

En el análisis realizado sobre los descriptores de las tesis en Educación Superior se observa una dispersión temática. Las temáticas en las que se centran las tesis doctorales son diversas tratando campos desde las Ciencias Económicas hasta Lingüística o Políticas Sociales. Si bien es cierto que la mayoría de ellas se encuentran relacionadas con el ámbito educativo y sus diferentes vertientes (formación profesional, métodos pedagógicos u organización y dirección de las instituciones educativas), predominando este ámbito como línea de investigación principal. Este hecho se ve reflejado en los departamentos en los que se enmarcan estas tesis, en los que sobresalen las áreas de Ciencias de la Educación y Ciencias Sociales. Aunque esta adscripción está bastante arraigada, lo cierto es que la Educación Superior es tratada desde otros campos en los que es objeto de estudio tal y como reflejan los departamentos y programas de doctorado a los que pertenecen como en economía, psicología, ciencias de la computación y tecnología informática, historia y arte, agricultura, ciencias de la tierra, derecho, matemáticas, medicina clínica y epidemiología, y tecnología electrónica y de las comunicaciones. Por lo tanto, el foco en el que han

Figura 6. Nube de palabras de los títulos de las tesis doctorales en Educación Superior

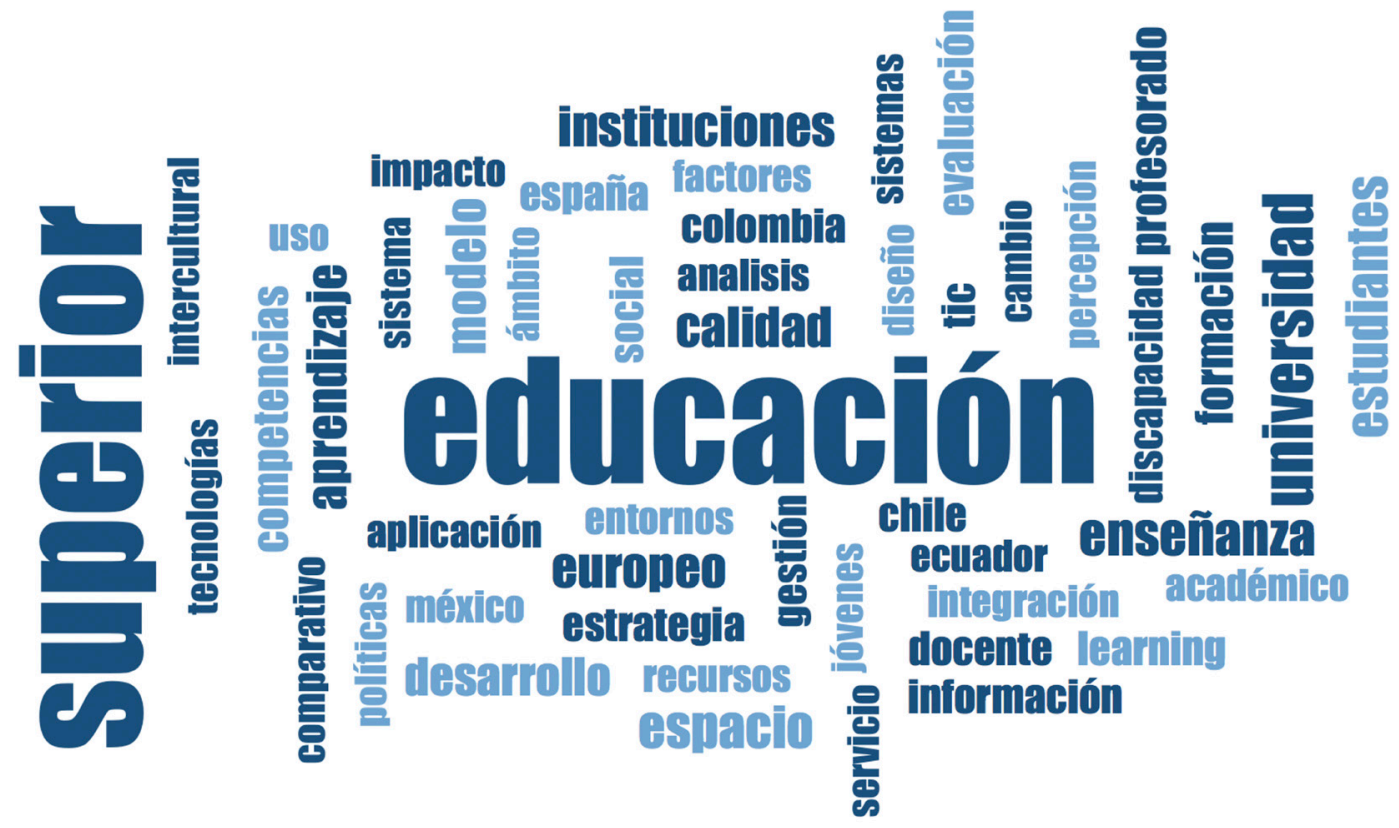


estado orientadas las investigaciones de los doctorandos aglutina diversos campos, convirtiéndose la Educación Superior en un campo transversal y multidisciplinar.

A pesar de que en otros estudios se ha constatado que la producción española de tesis doctorales en educación sigue un modelo cuasi-logístico con una preocupante tendencia de caída en los últimos doce años (Fernández-Bautista y otros, 2014), en el caso del estudio de la Educación Superior esta situación varía. La preocupación por estudiar este tema ha sido relativamente reciente. En concreto, nos encontramos con tesis doctorales en los años ochenta y noventa, pero es en la última década, a partir del año 2010, cuando se produce un aumento considerable de las mismas de forma progresiva. En los últimos años se han leído el $83 \%$ de las tesis doctorales con respecto a total. Este dato puede deberse a la necesidad de evaluar el proceso de transformación europeo, el movimiento e-learning y la importancia concedida a las competencias en TIC o el emprendimiento (Findler y otros, 2018; Puig y otros, 2019; Tight, 2018).

Las variables analizadas en el estudio ponen de manifiesto las diferencias entre hombres y mujeres en el ámbito académico. Aunque en la autoría y dirección de las tesis doctorales no hay diferencias significativas (ver Figura 4), sí que es destacable la escasa presencia de las mujeres en los tribunales de tesis, especialmente desarrollando el cargo de presidenta del mismo. Esta diferencia entre hombres y mujeres sigue siendo preocupante y de interés, puesto que el ámbito educativo ha contado especialmente con mayor presencia femenina (Salgado-Orellana y otros, 2018).

Se ha podido observar dentro de las tendencias en la producción científica de tesis doctorales en Educación Superior, cómo la co-dirección es un fenómeno creciente que se ha estabilizado en los últimos años. Este aumento puede deberse a dos motivos principalmente, por un lado, a cuestiones metodológicas y, por otro, a las limitaciones que la normativa impone a los Profesores Ayudantes Doctores a los que se les permite únicamente la co-dirección de las tesis doctorales. Este hecho es controvertido puesto que la valoración de la tesis doctoral como mérito es igual para ambos directores y está incluida en los baremos para la consecución de acreditaciones, así como para las plazas de profesorado, valorándose doblemente aquellas que han sido dirigidas únicamente por un único director, hecho limitado por la normativa para los Profesores Ayudantes Doctores (Olivera Zaldua y otros, 2016).
Los resultados de este estudio aportan mayor análisis en el campo de la bibliometría en tesis doctorales en un campo específico. Aunque la Educación sí que ha sido objeto de estudio en este tipo de análisis, la Educación Superior no se ha analizado de forma independiente mostrando su carácter multidisciplinar, su heterogeneidad en las áreas implicadas y la diferencia de géneros existente. A nivel universitario, este estudio permite conocer la evolución de la Educación Superior como ámbito de estudio y fundamentalmente saber cuáles son las principales líneas de investigación con mayor auge en la formación de doctorandos. Asimismo, se denotan diferencias de género en el campo educativo, como punto de preocupación en la igualdad de género en puestos de dirección y presidencia.

En síntesis, la Educación Superior como campo de estudio en la investigación doctoral ha sufrido un proceso de consolidación con un crecimiento lineal y constante en la producción de tesis doctorales. Sería interesante poder analizar esta tendencia en la publicación de artículos científicos en este sector, así como realizar un análisis de redes sociales al tratarse de un sector multidisciplinar.

Aunque la base de datos de TESEO contenga limitaciones como las expuestas anteriormente en relación a la exportación de datos (carencias a nivel descriptivo, dependencia de inclusión de información por parte de las universidades y/o doctorandos, entre otros), es la más completa en nuestro país en relación a las tesis doctorales defendidas, y nos permite conocer el perfil investigador del profesorado universitario, incluyendo los intereses de los doctorandos (Moyano y otros, 2006). Existen otras iniciativas que incluyen datos similares a TESEO, como son la base de datos de Tesis en Red, DIALNET, datos recogidos por el Instituto Nacional de Estadística, REBIUN, CISNE, Biblioteca Virtual Miguel de Cervantes o Mastesis. Sin embargo, estas fuentes en algunos casos se focalizan en universidades pertenecientes a una misma región y no incluyen la totalidad de campos detallados en TESEO, por lo que ésta se ha convertido en la base de datos de referencia en la realización de estudios de corte bibliométrico en esta materia.

En este artículo no se valora la calidad de la tesis, aunque como señala Díaz (Díaz, 2010), su calidad depende de la resolución de problemas prácticos que plantee, así como de su contribución al conocimiento científico, especialmente a través de las publicaciones científicas. En este sentido, habría que tener en cuenta la modalidad de tesis por compendio de publicaciones que las universidades españolas ofrecen en los últimos años. 


\section{REFERENCIAS}

Buela-Casal, G.; Guillén-Riquelme, A.; Ramiro-Sánchez, T.; Quevedo-Blasco, R. (2014). Ranking de investigación de las universidades públicas españolas. Revista Iberoamericana de Psicologia y Salud, 8 (1), 21-35. https://doi.org/10.23923/j.rips.2017.08.003

Buela-Casal, G.; Paz Bermúdez, M.; Sierra, J. C.; GuillénRiquelme, A.; Quevedo-Blasco, R. (2015). Productividad y eficiencia en investigación por comunidades autónomas españolas según la financiación (2012). Revista Iberoamericana de Psicologia y Salud, 6 (1), 1-10. https://doi.org/10.1016/S2171-2069(15)70001-3

De Lázaro y Torres, M. L. (2002). La Geografía a las puertas del tercer milenio a partir de las tesis doctorales leídas en los noventa. Anales de Geografía de La Univ. Complutense, 22, 49-66.

Delgado López-Cózar, E.; Torres-Salinas, D.; JiménezContreras, E.; Ruiz-Pérez, R. (2006). Análisis bibliométrico y de redes sociales aplicado a las tesis bibliométricas defendidas en España (1976-2002): temas, escuelas científicas y redes académicas. Revista Española de Documentación Científica, 29 (4), 493-524. https://doi.org/10.3989/redc.2006.v29.i4.306

Díaz Campo, J. (2014). La investigación sobre Internet en las facultades de comunicación española. Análisis bibliométrico de tesis doctorales (1997-2012). Documentación de Las Ciencias de La Información, 37, 305-320. https://doi.org/10.5209/rev_dcin.2014. v37.46828

Díaz Campo, J. (2018). Análisis bibliométrico de las tesis doctorales sobre Ética de los Medios de Comunicación presentadas en España (1979-2013). Doxa Comunicación. Revista Interdisciplinar de Estudios de Comunicación y Ciencias Sociales, 20, 65-88. https://doi. org/10.31921/doxacom.n20a3

Díaz, M. D. M. (2010). Evaluación y mejora de los estudios de Doctorado - PhD assessment and improvement. Revista de Educación, 352, 569-581.

Fernández-Bautista, A.; Torralbo, M. ; Fernández-Cano, A. (2014). Análisis longitudinal de tesis doctorales españolas en educación (1841-2012). RELIEVE - Revista Electronica de Investigacion y Evaluacion Educativa, 20 (2), 1-15. https://doi.org/10.7203/relieve.20.2.4479

Fernández-Guerrero, I. M. (2015). Tesis doctorales españolas en medicina de urgencias y emergencias (19782013). Emergencias, 27 (2), 129-134.

Ferreira-Villa, C.; Pascual-García, L.; Pol-Asmarats, C. (2013). La producción española en Tesis Doctorales sobre orientación en la Base de Datos TESEO (20012012). RELIEVE - Revista Electrónica de Investigación y Evaluación Educativa, 19 (1), 1-10. https://doi. org/10.7203/relieve.19.1.2460

Findler, F.; Schönherr, N.; Lozano, R.; Reider, D.; Martinuzzi, A. (2018). The impacts of higher education institutions on sustainable development: A review and conceptualization. International Journal of Sustaina- bility in Higher Education, 20 (1), 23-38. https://doi. org/10.1108/IJSHE-07-2017-0114

Fuentes Pujol, E.; Arguimbau Vivó, L. (2010). Las tesis doctorales en España (1997-2008): análisis, estadísticas y repositorios cooperativos. Revista Española de Documentación Científica, 33 (1), 63-89. https://doi. org/10.3989/redc.2010.1.711

Gallego Morón, N. (2016). Breaking the glass ceiling: The defense of the doctoral thesis as a key moment. Mètode Revista de Difusió de La Investigació, 91, 65-71. https://doi.org/10.7203/metode.7.8077

Gómez, E. L. (2016). Análisis de las tesis doctorales sobre tutoría: Aproximación bibliométrica y tendencias temáticas. Revista General de Informacion y Documentacion, 26 (1), 147-164. https://doi.org/10.5209/ rev_RGID.2016.v26.n1.53047

Hernández Pina, F.; Díaz Martínez, E. (2010). La formación de Doctores en el contexto del EEES. Una formación basada en competencias. Revista Fuentes, 10, 69-82.

Jiménez-Contreras, E.; Ruiz Pérez, R.; Delgado LópezCózar, E. (2014). El análisis de las tesis doctorales como indicador evaluativo: Reflexiones y propuestas. Revista de Investigacion Educativa, 32 (2), 295-308. https://doi.org/10.6018/rie.32.2.197401

Ley 14/2011, de 1 de junio, de la Ciencia, la Tecnología y la Innovación (Boletín Oficial del Estado, núm. 131, de 02/06/2011).

Moreno-Fernández, O.; Moreno-Crespo, P. (2016). Análisis bibliométrico de las tesis doctorales españolas indexadas con el descriptor "Sector de la educación" (1976/2014). Revista Española de Documentación Científica, 39 (3), e146. https://doi.org/10.3989/ redc. 2016.3 .1331

Moyano, M.; Domínguez, C. J. D.; Casal, G. B. (2006). Análisis de la productividad científica de la psiquiatría Española a través de las tesis doctorales en la base de datos TESEO (1993-2002). International Journal of Psychology and Psychological Therapy, 6 (1), 111-120.

Olivera Zaldua, M.; Sánchez Vigil, J. M.; Marcos Recio, J. C. (2016). Análisis de las tesis doctorales sobre fotografía en la universidad española (enero de 2013-marzo de 2016). Ibersid, 10 (2), 13-20.

Olmedilla, A. ; Abenza, L. ; Serrano, A., Muñoz, A. M.; García-Angulo, F.; Ortega, E. (2017). Estudio bibliométrico de tesis doctorales sobre psicología del deporte. Cuadernos de Psicología Del Deporte, 17 (2), 121-130.

Puig, B.; Blanco-Anaya, P.; Bargiela, I. M.; CrujeirasPérez, B. (2019). A systematic review on critical thinking intervention studies in higher education across professional fields. Studies in Higher Education, 44 (5), 860-869. https://doi.org/10.1080/030 75079.2019 .1586333 
Real Decreto 1393/2007, de 29 de octubre, por el que se establece la ordenación de las enseñanzas universitarias oficiales (Boletín Oficial del Estado, núm. 260, de 30/10/2007).

Real Decreto 99/2011, de 28 de enero, por el que se regulan las enseñanzas oficiales de doctorado (Boletín Oficial del Estado, núm. 35, de 10/02/2011).

Salgado-Orellana, N.; Ferrada, C.; Puraivan, E.; Parraguez, R. (2018). Un estudio cienciometrico sobre las tesis doctorales españolas en acción tutorial (1980-2016). Revista General de Información y Documentación, 28 (2), 415-434. https://doi.org/10.5209/rgid.62832
Sánchez Jiménez, R.; Blázquez Ochando, M.; Montesi, M.; Botezan, I. (2017). La producción de tesis doctorales en España (1995-2014): evolución, disciplinas, principales actores y comparación con la producción científica en WoS y Scopus. Revista Española de Documentación Científica, 40 (4), 1-17. https://doi.org/10.3989/ redc. 2017.4.1409

Tight, M. (2018). Systematic reviews and meta-analyses of higher education research. European Journal of Higher Education, 9 (2), 133-152. https://doi.org/10.108 $0 / 21568235.2018 .1541752$ 